\title{
Prophage Rs551 and Its Repressor Gene orf14 Reduce Virulence and Increase Competitive Fitness of Its Ralstonia solanacearum Carrier Strain UW551
}

\author{
Abdelmonim Ali Ahmad ${ }^{1,2}$, Michael J. Stulberg't and Qi Huang ${ }^{1 *}$ \\ ${ }^{1}$ Floral and Nursery Plants Research Unit, United States National Arboretum, United States Department of \\ Agriculture-Agricultural Research Service, Beltsville, MD, United States, ${ }^{2}$ Department of Plant Pathology, Faculty of \\ Agriculture, Minia University, El-minia, Egypt
}

\section{OPEN ACCESS}

Edited by:

William Michael McShan, University of Oklahoma Health Sciences Center, United States

Reviewed by:

Alessandra Occhialini, Université de Montpellier, France

Paul Hyman,

Ashland University, United States

*Correspondence:

Qi Huang

qi.huang@ars.usda.gov

tPresent address:

Michael J. Stulberg,

CPHST Beltsville Laboratory,

United States Department of Agriculture/Animal and Plant Health Inspection Service, Beltsville, MD, United States

\section{Specialty section:}

This article was submitted to

Virology,

a section of the journal

Frontiers in Microbiology

Received: 29 September 2017 Accepted: 29 November 2017

Published: 22 December 2017

Citation:

Ahmad AA, Stulberg MJ and Huang Q (2017) Prophage Rs551 and Its Repressor Gene orf14 Reduce Virulence and Increase Competitive Fitness of Its Ralstonia solanacearum

Carrier Strain UW551.

Front. Microbiol. 8:2480.

doi: 10.3389/fmicb.2017.02480
We previously characterized a filamentous lysogenic bacteriophage, $\phi R s 551$, isolated directly from the race 3 biovar 2 phylotype IIB sequevar 1 strain UW551 of Ralstonia solanacearum grown under normal culture conditions. The genome of $\phi R s 551$ was identified with $100 \%$ identity in the deposited genomes of 11 race 3 biovar 2 phylotype IIB sequevar 1 strains of $R$. solanacearum, indicating evolutionary and biological importance, and ORF14 of $\phi R s 551$ was annotated as a putative type-2 repressor. In this study, we determined the effect of the prophage and its ORF14 on the virulence and competitive fitness of its carrier strain UW551 by deleting the orf14 gene only (the UW551 orf14 mutant), and nine of the prophage's 14 genes including orf14 and six out of seven structural genes (the UW551 prophage mutant), respectively, from the genome of UW551. The two mutants were increased in extracellular polysaccharide production, twitching motility, expression of targeted virulence and virulence regulatory genes (pilT, egl, pehC, hrPB, and phcA), and virulence, suggesting that the virulence of UW551 was negatively regulated by $\phi R s 551$, at least partially through ORF14. Interestingly, we found that the wt $\phi$ Rs551-carrying strain UW551 of $R$. solanacearum significantly outcompeted the wt strain RUN302 which lacks the prophage in tomato plants co-inoculated with the two strains. When each of the two mutant strains was co-inoculated with RUN302, however, the mutants were significantly out-competed by RUN302 for the same colonization site. Our results suggest that ecologically, $\phi R s 551$ may play an important role by regulating the virulence of and offering a competitive fitness advantage to its carrier bacterial strain for persistence of the bacterium in the environment, which in turn prolongs the symbiotic relationship between the phage фRs551 and the R. solanacearum strain UW551. Our study is the first toward a better understanding of the co-existence between a lysogenic phage and its carrier plant pathogenic bacterial strain by determining the effect of the prophage Rs551 and its repressor on the virulence and competitive fitness of its carrier strain UW551 of R. solanacearum.

Keywords: Ralstonia solanacearum, filamentous lysogenic phage, prophage, phage repressor, competitive fitness, race 3 biovar 2, phylotype, sequevar 


\section{INTRODUCTION}

Bacterial wilt, a soil borne vascular disease caused by Ralstonia solanacearum, is one of the most devastating bacterial diseases in tropical, subtropical, and temperate regions of the world. The bacterium has a host range of over 450 plant species, including ornamentals such as geranium and economically important crops such as tomatoes and potatoes (Hayward, 1991; Kelman et al., 1994; Denny, 2006). R. solanacearum normally invades host plants from soil through wounds in roots, reproduces in the xylem vessels, and spreads rapidly through the plant's vascular system resulting in wilting and death of the plant (Vasse et al., 2000). The bacterium can be spread in soil or water or through latently infected plant materials like potato tubers or geranium cuttings.

Ralstonia solanacearum is historically classified into five races and five biovars based on host range and biochemical properties, respectively. Molecular classification, however, has thus far grouped $R$. solanacearum into 4 phylotypes and 53 sequevars (Albuquerque et al., 2014; Stulberg and Huang, 2016). The race 3 biovar 2 (phylotype IIB sequevars 1 and 2) of $R$. solanacearum causes devastating brown rot of potato, and is a quarantine pathogen in many countries and listed as a select agent in the United States (Agricultural Bioterrorism Protection Act of 2002, 2002).

Biochemical and molecular genetic studies over the past 60 years have explored the underlying mechanisms of $R$. solanacearum pathogenesis. Several major virulence factors have been identified, including extracellular polysaccharides (EPSs), plant cell wall degrading enzymes such as the $\beta$-1,4-endoglucanase (Egl) (Roberts et al., 1988), endopolygalacturonase (PehA) (Schell et al., 1988; Allen et al., 1991), and exopolygalacturonases (PehB and PehC) (Huang and Allen, 2000; González and Allen, 2003), as well as type IV pili (Tfp) that is essential for twitching motility, adherence and colonization, and biofilm formation (Liu et al., 2001; Kang et al., 2002). The expression of these virulence genes is controlled by PhcA, a LysR-type global transcriptional regulator (Schell, 2000). $R$. solanacearum also uses a key transcriptional activator, HrpB, to drive the expression of $h r p$ genes encoding a type III secretion system and effector molecules that allow translocation of the effector proteins into plant cells for pathogenicity (Genin and Boucher, 2004; Delaspre et al., 2007).

Control of bacterial wilt is mainly achieved by exclusion and eradication. For the past 10 years, a wide range of lysogenic and lytic bacteriophages specifically infecting $R$. solanacearum have been isolated from soil of crop fields, and their use as potential biocontrol agents has been explored (Yamada et al., 2007; Fujiwara et al., 2011; Bhunchoth et al., 2015). We recently isolated a filamentous lysogenic bacteriophage $\phi$ Rs551 directly from the race 3 biovar 2 strain UW551 of $R$. solanacearum grown under normal culture conditions (Ahmad et al., 2017). The phage has a particle size of about $1,200 \mathrm{~nm}$ in length and $7 \mathrm{~nm}$ in width, and has a genome size of 7,929 nucleotide with 14 open reading frames (Ahmad et al., 2017). In contrast with other R. solanacearum phages isolated from soil, $\phi$ Rs551 is the first isolated phage that contains a resolvase (ORF13) and a putative type-2 phage repressor (ORF14), although how this repressor maintains a prophage state and phage immunity in $\phi$ Rs551 had not been studied. In addition, the genome sequence of $\phi$ Rs551 is surprisingly found with $100 \%$ identity in the deposited genomes of 11 race 3 biovar 2 phylotype IIB sequevar 1 strains of $R$. solanacearum, indicating evolutionary importance (Ahmad et al., 2017). Infection of a susceptible $R$. solanacearum strain RUN302 by $\phi$ Rs551 resulted in colonies with less fluidal appearance and reduced EPS production, motility, and virulence (Ahmad et al., 2017). It is unclear, however, what effects the phage or the putative phage repressor has on its host strain R. solanacearum UW551.

Huerta et al. (2015) recently hypothesized that differences in temperature adaptation and competitive fitness account for the uneven geographic distribution of $R$. solanacearum strains, and found that lowland tropical and warm temperate strains out-compete temperate strains of $R$. solanacearum, probably due to bacteriocins produced by the tropical and warm temperate strains to specifically inhibit the growth of the temperate strains. $R$. solanacearum lytic phages encode bacteriolytic proteins (Ozawa et al., 2001) and are lytic to susceptible strains (Fujiwara et al., 2011), and lysogenic phages are known to bring novel phenotypic properties that might affect the fitness of their host bacteria (Casjens, 2003; Brüssow et al., 2004; Canchaya et al., 2004; Davies et al., 2016). We therefore hypothesize that $R$. solanacearum strains prevalent under different environmental conditions may contain different phages that offer competitive fitness to their host strain, allowing the host strains to persist in the environment by preventing the establishment of susceptible strains of $R$. solanacearum that lack the lysogen.

To better understand the contribution of the putative type2 repressor of $\phi$ Rs551 and the phage $\phi$ Rs551 itself to the virulence and competitive fitness of its carrier strain UW551 of $R$. solanacearum, we generated two $R$. solanacearum mutants by knocking out the orf14 gene, and nine of the 14 genes of $\phi$ Rs551 from the prophage region of UW551, respectively. The phenotype of the two mutants included increased EPS production and twitching motility, increased expression of five other genes tested, as well as increased virulence when inoculated into tomato plants alone. When co-inoculated with strain RUN302 which lacks the prophage Rs551 in its genome for infection of tomato plants, these mutants also had decreased competitive fitness in colonizing tomato stems and had little effect on the virulence of strain RUN302.

\section{MATERIALS AND METHODS}

\section{Bacterial Strains and Plasmids}

Bacterial strains and plasmids used and constructed in this study are listed in Table 1.

\section{Growth and Isolation of Bacterial Strains}

Ralstonia solanacearum was grown and its inocula prepared as described (Stulberg et al., 2015). To isolate $R$. solanacearum from inoculated plant samples, $0.5-\mathrm{cm}$ plant stem sections were prepared and homogenized as described (Stulberg and Huang, 
TABLE 1 | Bacterial strains and plasmids used in this study.

\begin{tabular}{|c|c|c|}
\hline Designation & Relevant characteristics ${ }^{a}$ & Source or reference \\
\hline \multicolumn{3}{|l|}{ Strains } \\
\hline \multicolumn{3}{|l|}{ Ralstonia solanacearum } \\
\hline UW551 & Wild-type, race 3 biovar 2, phylotype IIB sequevar 1, $\phi$ Rs551 carrier & C. Allen, United States \\
\hline UW551 $\Delta$ фRs551-orf14 (UW551 orf14 mutant) & $\begin{array}{l}\text { UW551 with a 342-bp prophage region containing orf14 replaced with a } \\
\text { 616-bp Gm cassette, Gm } \mathrm{R}\end{array}$ & This study \\
\hline UW551 & $\begin{array}{l}\text { UW551 with a 3,321-bp prophage region including orf14 and orf1 to orf8 } \\
\text { replaced with a 616-bp Gm cassette, GmR }\end{array}$ & This study \\
\hline RUN302 & Wild-type, biovar 1, phylotype IIB sequevar 4, $\phi R s 551^{S}$ & P. Prior, France \\
\hline \multicolumn{3}{|l|}{ Escherichia coli } \\
\hline TOP10 & $\begin{array}{l}\text { F- mcrA } \Delta(\text { mrr-hsdRMS-mcrBC) } \text { Ф80lacZ } \Delta \mathrm{M} 15 \Delta \text { lacX74 recA1 araD139 } \\
\Delta(\text { ara-leu)7697 ga/U galK rpsL (StrR) endA1 nupG }\end{array}$ & Invitrogen \\
\hline TP997 & MG1655 lacIPA::bla-aadA1148 galK::aacC1067 & Addgene \\
\hline \multicolumn{3}{|l|}{ Plasmid } \\
\hline pCR ${ }^{\text {TM }}$ Blunt II TOPO & PCR cloning vector, $\operatorname{Kan}^{R} Z_{c^{R}}$ & Invitrogen \\
\hline pCR Blunt II-Gm & pCR $^{\text {TM }}$ Blunt II TOPO with a 616-bp Gm cassette, $\mathrm{Gm}^{R} \mathrm{Kan}^{R} \mathrm{Zc}^{R}$ & This study \\
\hline pCR Blunt II- $\phi$ Rs551-orf14 Up-Gm- $\phi$ Rs551-orf14 Down & $\begin{array}{l}\text { pCR Blunt II-Gm with 994-bp upstream and 819-bp downstream fragments of } \\
\text { the 342-bp prophage region in } R \text {. solanacearum inserted before and after the } \\
\text { Gm cassette, respectively, } \mathrm{Gm}^{\mathrm{R}} \mathrm{Kan}^{\mathrm{R}} \mathrm{Zc}^{\mathrm{R}}\end{array}$ & This study \\
\hline pCR Blunt II- $\phi$ Rs551 Up-Gm- $\phi$ Rs551 Down & $\begin{array}{l}\text { pCR Blunt II-Gm with 994-bp upstream and 850-bp downstream fragments of } \\
\text { the 3,321-bp prophage region in } R \text {. solanacearum inserted before and after the } \\
\text { Gm cassette, respectively, } \mathrm{Gm}^{R} \operatorname{Kan}^{R} \mathrm{Zc}^{R}\end{array}$ & This study \\
\hline
\end{tabular}

$\mathrm{a}_{\mathrm{Gm}}{ }^{R}, \mathrm{Kan}^{R}$, and $\mathrm{Zc}^{R}$ indicate resistance to gentamicin, kanamycin, and zeocin, respectively.

2015), and dilution plated onto modified semi-selective medium agar plates (Huang and Lakshman, 2010). Escherichia coli strains were cultured at $37^{\circ} \mathrm{C}$ in Luria-Bertani medium (Miller, 1972). When needed, antibiotics were added at $25 \mu \mathrm{g} / \mathrm{ml}$ for kanamycin and $15 \mu \mathrm{g} / \mathrm{ml}$ for gentamicin. Since $R$. solanacearum strain UW551 is a select agent pathogen in the United States, manipulation of the strain was conducted in a secured laboratory and virulence assays described below were performed in a secured greenhouse section approved for select agent research by USDA/APHIS using standard operating procedures also approved by APHIS for race 3 biovar 2 strains of $R$. solanacearum.

\section{DNA Isolation and Manipulation}

Standard molecular biology techniques were used for plasmid isolation, restriction digestion, cloning, and transformation of E. coli strains (Sambrook and Russell, 2001). Total bacterial DNA was extracted using Qiagen's Blood and Tissue Kit (Qiagen, Chatsworth, CA, United States) following the manufacturer's instructions.

\section{Design of PCR Primers and PCR Conditions}

Primers designed in this study were listed in Table 2. They were designed based on the deposited UW551 draft genome sequence in GenBank (ASM16795v1, GCA_000167955). The regions selected for primer design were entered into the free online A plasmid Editor (ApE) program. Similar design parameters $\left(\mathrm{GC}=45-60 \%, \mathrm{Tm}=60-64^{\circ} \mathrm{C}\right.$, primer length $\left.18-26\right)$ were used for primers in each pair. The specificity of each primer pair and amplicon was checked by BLASTn against the UW551 genome, and the nr and WGS databases in GenBank for specificity.
Colony PCR was performed by picking $R$. solanacearum cells using a sterile toothpick or pipette tip from a single colony grown on a plate and mixing the cells in $100 \mu \mathrm{l}$ of sterile water. The cell suspension was boiled for $5 \mathrm{~min}$ and cooled on ice or stored at $-20^{\circ} \mathrm{C}$ until use. Two to five microliters of the suspension were used for PCR.

PCR to amplify the upstream and downstream prophage regions was conducted in a $20 \mu$ l volume containing $1 x$ KAPA HiFi HotStart ReadyMix (Kapa Biosystems, Boston, MA, United States), 5 pmol of each primer, and approximately $20 \mathrm{ng}$ of DNA template. PCR conditions were 1 cycle of $3 \mathrm{~min}$ at $95^{\circ} \mathrm{C}$, followed by 30 cycles of $20 \mathrm{~s}$ at $98^{\circ} \mathrm{C}, 15 \mathrm{~s}$ at $58^{\circ} \mathrm{C}$, and $30 \mathrm{~s}$ at $72^{\circ} \mathrm{C}$, with a final extension of $2 \mathrm{~min}$ at $72^{\circ} \mathrm{C}$. PCR to amplify virulence-related genes was conducted in a $20-\mu l$ volume containing 1x GoTaq Green Master Mix (Promega, Madison, WI, United States), $20 \mathrm{ng}$ of template DNA, and $5 \mathrm{pmol}$ of each primer. PCR conditions were 1 cycle of $4 \mathrm{~min}$ at $94^{\circ} \mathrm{C}, 30$ cycles of $1 \mathrm{~min}$ at $94^{\circ} \mathrm{C}, 1 \mathrm{~min}$ at $60^{\circ} \mathrm{C}$, and $1 \mathrm{~min}$ at $72^{\circ} \mathrm{C}$, with a final extension of $10 \mathrm{~min}$ at $72^{\circ} \mathrm{C}$.

\section{Construction of $R$. solanacearum Mutants}

To study the role of orf14 of the prophage Rs551 and the prophage itself in $R$. solanacearum, two mutants of $R$. solanacearum which had deletions in the prophage were constructed in strain UW551 by homologous double recombination. Mutant UW551 $\Delta \phi$ Rs551-orf14, designated as the UW551 orf14 mutant, was generated by replacing a 342 -bp prophage region, located in contig 0570 of UW551 (GenBank accession number: AAKL01000012.1), with a 616-bp gentamicin cassette. The 342-bp fragment contained the entire 291-bp prophage region 
TABLE 2 | List of primers designed in this study.

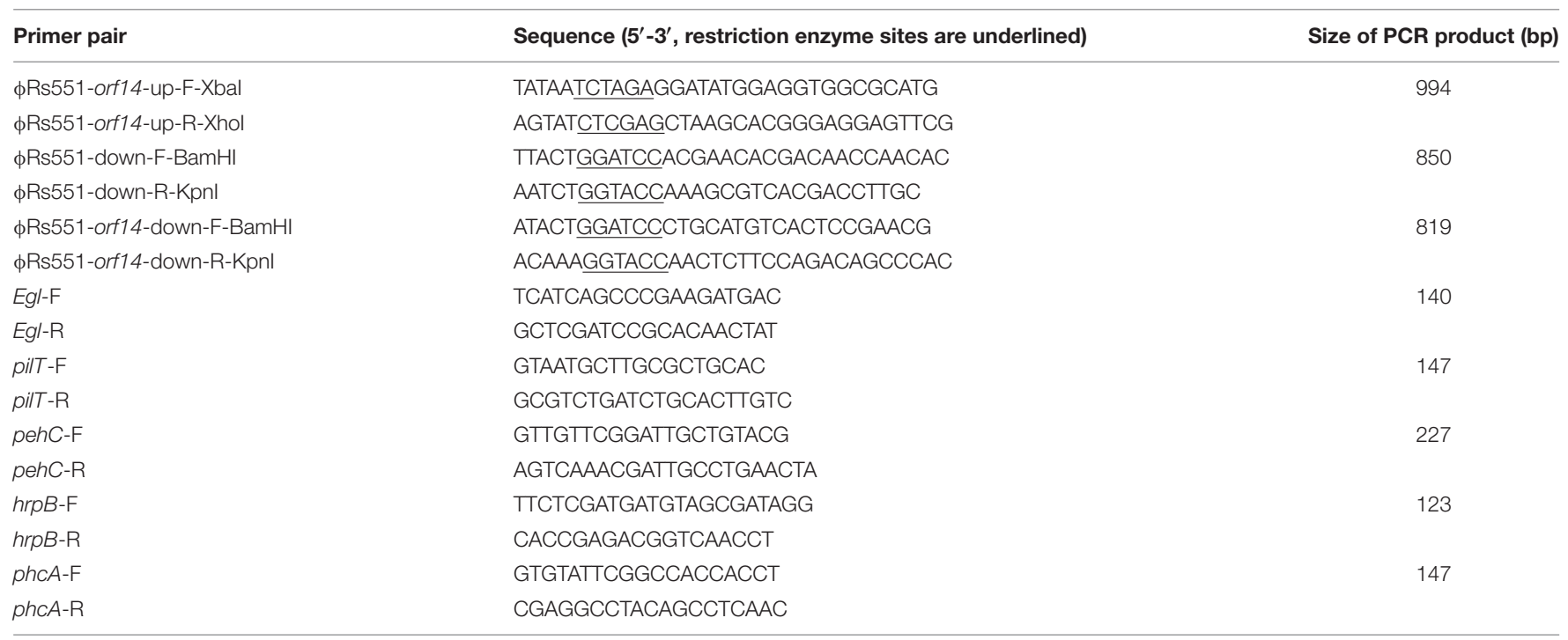

corresponding to $\phi$ Rs551's orf14, coding for a putative type-2 phage repressor, as well as 42-bp upstream and 9-bp downstream of orf14. Mutant UW551 $\Delta \phi$ Rs551, designated as the UW551 prophage mutant, was constructed by replacing a 3,321-bp prophage region with a 616-bp gentamicin cassette. The 3,321-bp fragment contained the same 342-bp in the orf14 mutant, as well as an additional 2,979-bp located in the same contig of UW551, corresponding to $\phi$ Rs551's orflin the replication module, orf 2 between the replication and structure modules, and six (orf3 to orf7, and 1,005-bp of the 1,524-bp of orf8) of the seven structural genes in the structural module of the prophage (Figure 1). To make the mutants, a 616-bp gentamicin cassette was first amplified from a colony of TP997, purchased from Addgene (Cambridge, MA, United States), by PCR with primers $5^{\prime}$-CGAATCCATGTGGGAGTTTA- $3^{\prime}$ and $5^{\prime}$ TTAGGTGGCGGTACTTGGGT-3' (Poteete et al., 2006). The cassette was then cloned into the TOPO site of the vector pCR Blunt II TOPO to generate pCR Blunt II-Gm using Invitrogen's Zero Blunt ${ }^{\circledR} \mathrm{TOPO}^{\circledR}$ PCR Cloning Kit according to the manufacturer's instructions. Two regions of DNA, 994-bp in size located upstream and 815-bp downstream of the 342-bp prophage region, were amplified by PCR using primers in Table 2, digested with respective restriction enzymes, and cloned sequentially into the multiple cloning sites before and after the gentamicin cassette in pCR Blunt II-Gm to obtain pCR Blunt II$\phi$ Rs551-orf14 Up-Gm- $\phi$ Rs551-orf14 Down. Similarly, the same 994-bp upstream fragment and a 850-bp downstream fragment of the 3,321-bp prophage region was cloned sequentially to obtain pCR Blunt II- $\phi$ Rs551 Up-Gm- $\phi$ Rs551 Down. The resulting plasmids were electroporated into competent cells of $R$. solanacearum strain UW551 as described by Ahmad et al. (2017). This was followed by selection on gentamicincontaining triphenyltetrazolium chloride (TZC) plates (Kelman, 1954) for transformants that had undergone homologous double recombination between the Up-Gm-Down region in pCR Blunt II-Up-Gm-Down and the Up and Down prophage sequences in the chromosome of UW551 of $R$. solanacearum. The knock-out mutants were identified by screening on TZC plates amended with gentamicin, and confirmed by PCR using primer pairs located within the sequences of the mutated regions that showed a lack of any amplified products. To determine if a phage was still produced from the UW551 mutants, an aliquot from the supernatant of the $R$. solanacearum mutants was subjected to the spot test and plaque-forming assay (Ahmad et al., 2017) using $R$. solanacearum RUN 302, a strain susceptible to $\phi$ Rs551 and which contained no $\phi$ Rs551 sequence in its genome before infection. The presence or absence of phage particles in the supernatant of the $R$. solanacearum mutants was also examined under transmission electron microscope (Ahmad et al., 2017).

\section{EPS, in Vitro Growth, and Twitching Motility Assays}

Extracellular polysaccharide in the supernatant of $R$. solanacearum was determined quantitatively, in vitro growth of $R$. solanacearum strains were measured, and twitching motility examined as described by Ahmad et al. (2017), except that twitching motility was visualized using a Zeiss AxioZoom v16 stereo zoom microscope (Carl Zeiss Microscope GmbH, Germany). Two replicates were used for each strain in the EPS assay and the experiment was repeated three times.

\section{RNA Isolation and Analysis of Gene Expression}

Total bacterial RNA was isolated from $3 \mathrm{ml}$ of $R$. solanacearum culture at the exponential growth phase $\left(\mathrm{OD}_{600}=0.3\right)$ using Qiagen's RNeasy Protect Bacterial and RNeasy Mini Kits (Qiagen, Inc., CA) according to the manufacturer's protocol. Ambion ${ }^{\circledR}$ TURBO DNA-free ${ }^{\mathrm{TM}}$ DNase Treatment and Removal Reagents (Life Technology) were used to remove contaminating DNA from the RNA preparation and to subsequently remove the DNase and divalent cations from the sample. The absence of DNA contaminants was confirmed by PCR using gene-specific primers (Table 2) on the RNA samples. The bacterial genomic DNA of 
R. solanacearum strain UW551 was used as positive, and sterile water negative controls. The RNA samples were quantified using the Nanodrop ND-1000 spectrophotometer (NanoDrop Tech. Inc.). One microgram of the RNA was reverse transcribed using Quantabio's qScript ${ }^{\mathrm{TM}}$ cDNA SuperMix (Quantabio, Beverly, MA, United States) according to the manufacturer's instructions. Quantitative PCR analysis was carried out with gene-specific primers (Table 2) using $1 \mu \mathrm{l}$ of cDNA as a template in a $20 \mu \mathrm{l}$ volume containing $10 \mu \mathrm{l}$ of $\mathrm{IQ}^{\mathrm{TM}} \mathrm{SYBR}^{\circledR}$ Green Supermix (Bio$\mathrm{Rad}$ ) and $0.5 \mu \mathrm{M}$ each of the gene primers. Cycling conditions were $95^{\circ} \mathrm{C}$ for $3 \mathrm{~min}$, followed by 45 cycles of $95^{\circ} \mathrm{C}$ for $10 \mathrm{~s}$, $60^{\circ} \mathrm{C}$ for $15 \mathrm{~s}$, and $72^{\circ} \mathrm{C}$ for $30 \mathrm{~s}$. At the end of the program, melting curve $\left(65-95^{\circ} \mathrm{C}\right.$ with a heating rate of $\left.0.5^{\circ} \mathrm{C} / \mathrm{min}\right)$ was analyzed to confirm the specificity of the primer set (Addy et al., 2012a). Relative levels of gene expression were determined using the $2^{-\Delta \Delta C_{\mathrm{T}}}$ method (Livak and Schmittgen, 2001), with the $16 \mathrm{~s}$ rRNA gene as the internal control (Addy et al., 2012a). The experiments were performed three times with two replicates each time.

\section{Virulence and Competition Assays}

For virulence assays, tomato plants were seeded, transplanted, inoculated by soil drenching with $50 \mathrm{ml}$ of $R$. solanacearum $\left(5 \times 10^{7}\right.$ cells $\left./ \mathrm{ml}\right)$ (Ahmad et al., 2017). Inoculated plants were rated using a disease index (DI) ranging from 0 (healthy) to 4 (76-100\% leaves wilted) (Roberts et al., 1988). There were 10 plants per treatment and the experiment was repeated three times. To determine if $\phi R 5551$ 's orf14 or the phage itself offers any competitive advantage to its carrier strain UW551, a competition assay was similarly performed as described for the virulence assay, except that in addition to inoculation with the $\phi$ Rs551susceptible strain RUN302 alone, tomato plants were also coinoculated with the following two different $R$. solanacearum strains, respectively, in a 1:1 $(25 \mathrm{ml}: 25 \mathrm{ml})$ ratio: (1) wt RUN302 (does not carry and is susceptible to $\phi$ Rs551) and the wt UW551 ( $\phi$ Rs551-carrier), (2) RUN302 and the UW551 orf14 mutant strain, and (3) RUN302 and the UW551 prophage mutant strain. Tomato plants inoculated with water were served as negative controls. As soon as the inoculated plants showed signs of wilting, bacterial colonies were isolated as described above. To estimate the ratio of and to differentiate between the two strains in the stem sections, 50 randomly picked colonies, 10 from each of five different wilted plants per treatment, were subjected to the multiplex PCR developed by Stulberg et al. (2015). The experiment was performed three times.

\section{Statistical Analysis}

Data for EPS dry weight and virulence were analyzed by one-way ANOVA using web-based statistical software ${ }^{1}$. Means were compared using the Tukey's Honest Significant Difference test provided by the software. The values in gene expression and competitive fitness were shown as the means of three experiments. Differences were considered statistically significant at $P<0.01$.

\footnotetext{
${ }^{1}$ http://vassarstats.net/anovalu.html
}

\section{RESULTS}

\section{Confirmation of $\boldsymbol{R}$. solanacearum Mutants and Determination of Phage Production by the Mutant Strains}

The 7,929-nucleotide genome sequence of $\phi$ Rs551 corresponds to nucleotides $73,039-80,967$ in contig 0570 of the deposited genome sequence of $R$. solanacearum strain UW551 (Figure 1). $R$. solanacearum mutants were confirmed by their ability to grow on TZC plates containing gentamicin, and by PCR for the absence of the 342-bp prophage region in the UW551 orf14 mutant and the 3,321-bp region in the UW551 prophage mutant (data not shown). The UW551 orf14 mutant was found to produce phage particles spontaneously in its supernatant at a rate similar to the wt strain UW551. This was shown when the supernatant of the overnight culture of the UW551 orf14 mutant strain was subjected to the spot test and plaque-forming assay using $\phi$ Rs551-suceptable strain $R$. solanacearum RUN302, similar plaque formation was observed, and a similar number of plaques was obtained as with the supernatant of the wt UW551 (data not shown). On the contrary, no plaques were formed when the supernatant of the prophage mutant strain was subjected to the same phage susceptibility assay, and no phage particles were observed under transmission electron microscope.

\section{Physiological Changes Were Detected in R. solanacearum Mutants}

To characterize the UW551 orf14 and prophage mutant strains, we first compared the in vitro growth of the mutants with their wt strain UW551, and found all three strains grew at a similar rate (data not shown). When the three strains grew on regular TZC medium plates, however, the colonies of the mutant strains appeared more fluidal and irregular than those of the wt UW551, suggesting a high production of EPS. This observation was confirmed by an EPS quantitative assay that showed both mutant strains produced significantly higher amounts of EPS (73.6 $\pm 4.5 \mathrm{mg} / 10 \mathrm{ml}$ for UW551 prophage mutant, and $59.6 \pm 9.5 \mathrm{mg} / 10 \mathrm{ml}$ for UW551 orf14 mutant) than the wt strain $(44.3 \pm 7.2 \mathrm{mg} / 10 \mathrm{ml})$. The difference in EPS production between the two mutant strains was not significant. The two mutant strains also displayed distinctly different twitching motility when compared with the wt strain UW551 (Figure 2). For the wt strain, we observed twitching motility under a microscope as indicated by the formation of corrugated trajectories with smooth edge around the margin of its colonies (Figure 2, left). The size of the trajectories, however, was larger with irregular edges in $R$. solanacearum mutant strains, especially in the orf14 mutant (Figure 2, middle).

\section{The UW551 orf14 and Prophage Mutant Strains of $R$. solanacearum Were More Virulent than the wt Strain UW551}

To study the effect of deletion of the targeted prophage regions in the virulence of $R$. solanacearum, we compared the virulence of the wt to that of the mutant strains of $R$. solanacearum (Figure 3 ). 


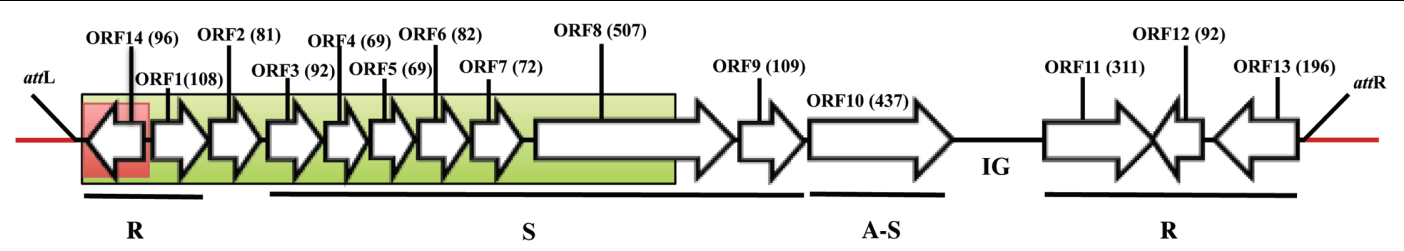

FIGURE 1 | Genomic organization of $\phi$ Rs551's identical prophage region in $R$. solanacearum strain UW551. Bacterial sequence is in red and the prophage sequence in black. The regions in red and green boxes were replaced with a 616-bb gentamicin cassette in UW551's orf14 mutant and prophage mutant strains, respectively. The open reading frames (ORF) in the prophage are represented by arrows with indicated direction of transcription, and the number of amino acids in each ORF is indicated. R, S, and A-S represent functional modules for replication, structure, and assembly and secretion, respectively. IG represents intergenic region. att $L$ and attR indicate the location of $\phi R s 551$ 's left and right attachment sites, respectively.

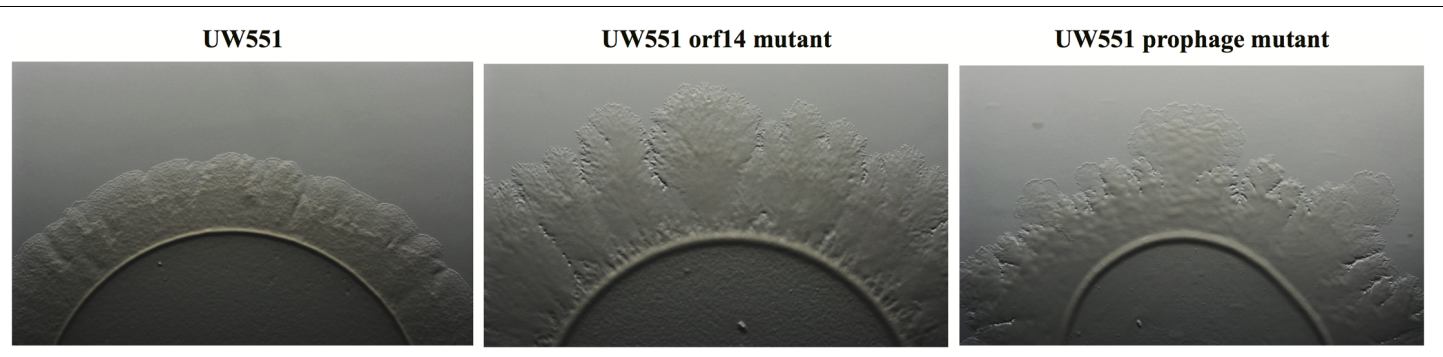

FIGURE 2 | Comparison of twitching motility of $R$. solanacearum strains UW551 (left), UW551 orf14 mutant (middle), and UW551 prophage mutant (right). Three microliters of bacterial suspension $\left(10^{8} \mathrm{cells} / \mathrm{ml}\right)$ were placed in the center on a minimal medium plate, and kept for $5 \mathrm{days}$ at $28^{\circ} \mathrm{C}(\mathrm{Ahmad}$ et al., 2017$)$. Visualization of twitching motility was done by placing the plate without its lid on the stage of a Zeiss AxioZoom v16 stereo zoom microscope (Carl Zeiss Microscope $\mathrm{GmbH}$, Germany) (Ahmad et al., 2017). Corrugated trajectories formed around colonies indicate twitching motility. Note that the edge of the corrugated trajectories was smooth in UW551, but not in the two mutant strains of $R$. solanacearum.

The wt strain UW551 did not cause any disease symptoms until 8 days after soil drenching inoculation (DI $>0$ ), and reached a DI of 3.1 at day 21 (Figure 3). The virulence level caused by the two mutant strains of $R$. solanacearum, however, was significantly higher (Figure 3). The mutants started to cause disease symptoms

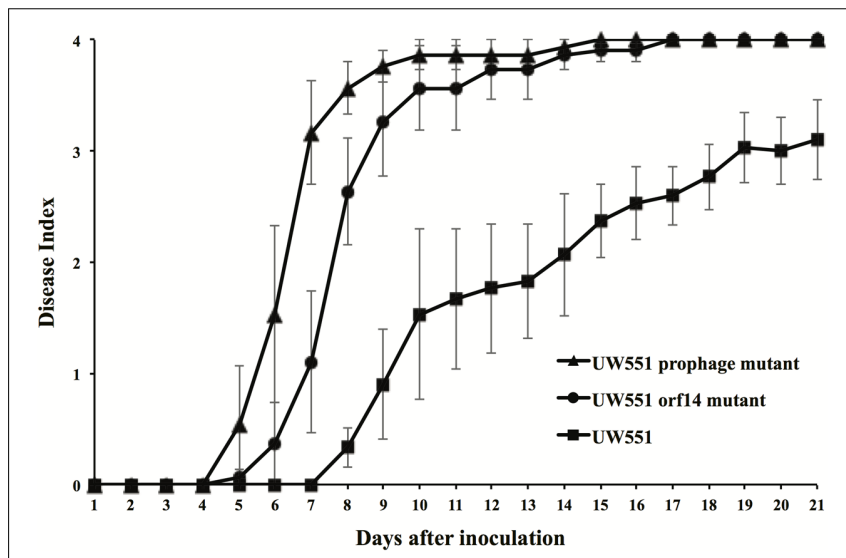

FIGURE 3 | Virulence of $R$. solanacearum strains. Two- to three-week-old tomato plants were inoculated by soil drenching with $50 \mathrm{ml}\left(5 \times 10^{7} \mathrm{cells} / \mathrm{ml}\right)$ of wt UW551 (squares), UW551 orf14 mutant (circles) or UW551 prophage mutant (triangles). Plants were rated using a DI of 0 (no wilting) to 4 (76-100\% leaves wilted). Points represent means of three separate experiments with 10 plants in each experiment for a total of 30 plants. Bars indicate standard errors.
5 days after inoculation and completely wilted all inoculated plants (DI $=4$ ) by day 17 (Figure 3). DIs caused by the UW551 prophage mutant were statistically similar to the ones caused by the UW551 orf14 mutant, except at days 7 and 8 (Figure 3).

\section{Gene Expression Levels Were Increased in $R$. solanacearum Mutant Strains}

To identify the factors contributing to increased virulence of the $R$. solanacearum mutant strains, expression of five genes (pilT, egl, pehC, $h r p B$, and $p h c A$ ), all known virulence factors of $R$. solanacearum, was compared between the mutant and the wt strains. The expression of all five genes was increased in the two mutant strains, with the pilT gene showing the greatest increase: 8 -fold in the orf 14 mutant and 21 -fold in the prophage mutant (Figure 4). The level of expression of the egl, pehC, $h r p B$, and phcA genes was increased between 2.0- and 4.5-fold in the two mutants (Figure 4).

\section{R. solanacearum orf14 and Prophage Mutant Strains of UW551 Are Out Competed by the Phage $\phi$ Rs551-Susceptible Strain RUN302 in} Plants

The effect of UW551 and its mutant strains on plant colonization by RUN302, a strain of $R$. solanacearum lacking the prophage Rs551, was studied. RUN302 was co-inoculated with one of the UW551 strains for infection of tomato plants. The ratio of the 


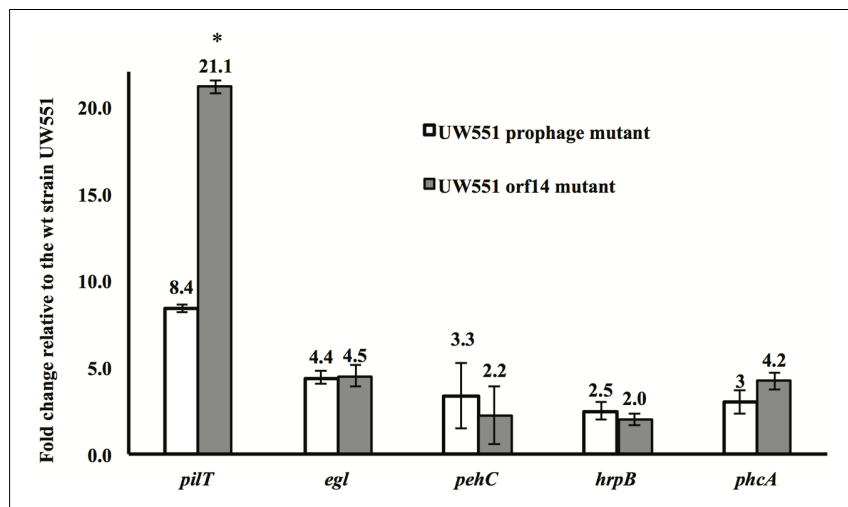

FIGURE 4 | Expression of the pilT, egl, pehC, hrpB, and phcA genes in $R$. solanacearum UW551 orf14 and prophage mutants, as compared to the wt strain UW551. Quantitative reverse transcription PCR from RNA extracted from $R$. solanacearum strains was performed to determine transcript levels of each gene. Each gene expression was calculated using the $2^{-\Delta \Delta C_{T}}$ method (Livak and Schmittgen, 2001) and normalized using the 16s rRNA gene as an internal control. Values shown are means of three separate experiments, each containing two replicates. Bars indicate standard errors. *indicates significant difference $(p<0.05)$ between the two mutants by the Student's $t$-test.

two mixed strains was determined in stem sections of infected tomato plants. When the wt $\phi$ Rs551-carrier strain UW551 was co-inoculated with the wt $\phi$ Rs551-lacking strain RNN302, 30 out of 50 randomly picked bacterial colonies isolated from the tomato stems belonged to UW551 (Figure 5), significantly more than the number of RUN302 colonies. On the contrary, when each of the two UW551 mutant strains was co-inoculated with RUN302, only 10 and 19 out of 50 were colonies of the prophage mutant strain and the orf14 mutant strain, respectively, significantly less than the number of RUN302 colonies in the stems (Figure 5).

To study the effect of UW551 and its mutant strains on the virulence of $R$. solanacearum strain RUN302, tomato plants were inoculated by soil drenching with RUN302 alone, or together (1:1) with the wt UW551, the UW551 orf14 mutant or the UW551 prophage mutant, respectively (Figure 6). When RUN302 was coinoculated with UW551, the co-inoculation caused a delayed and significantly lower DI 4 days after inoculation than inoculation with RUN302 alone (Figure 6). On the contrary, when RUN302 was co-inoculated with the UW551 prophage mutant, its overall virulence was similar to that caused by RUN302 alone (Figure 6). DIs caused by RUN302 co-inoculated with the UW551 orf14 mutant strain were lower than the ones caused by RUN302 alone or co-inoculation with RUN302 and the UW551 prophage mutant, but the difference was not significant 9 days after inoculation and all inoculated plants were completely wilted 6 days after that (Figure 6).

\section{DISCUSSION}

Currently, a wide range of $R$. solanacearum phages have been reported including filamentous phages of the family Inoviridae (Yamada et al., 2007; Murugaiyan et al., 2011; Van et al., 2014), and icosahedral phages of the families Myoviridae (Yamada et al., 2007; Bhunchoth et al., 2015), Podoviridae (Kawasaki et al., 2009, 2016; Bhunchoth et al., 2015), and Siphoviridae (Thi et al., 2015). Comparative genomics also revealed that $R$. solanacearum genomes contain many prophages of the families Inoviridae and Myoviridae (Yamada et al., 2007; Murugaiyan et al., 2011; Ahmad et al., 2017). The co-evolution between bacteria and bacteriophages plays a key role in driving and maintaining the ecology and evolution of microbial populations, and phages are known to change competitive dynamics among bacterial strains or species (Bohannan and Lenski, 2000a,b; Joo et al., 2006; Koskella et al., 2012; Koskella and Brockhurst, 2014). Temperate phages like the SMP phage of Streptococcus suis and the prophages of Pseudomonas aeruginosa affect their carrier bacteria in many ways (e.g., growth rate for the former and competitiveness for the latter), contributing to the fitness and virulence of the bacteria (Davies et al., 2016). Previous studies of $R$. solanacearum phages, however, had been focused exclusively on genomic characterization, integration mechanism, effect on their susceptible $R$. solanacearum strains and potential as biocontrols (Kawasaki et al., 2007a,b; Yamada et al., 2007; Askora et al., 2009, 2011; Addy et al., 2012a,b). Our study is the first toward a better understanding of the co-existence between a lysogenic phage and its carrier plant pathogenic bacterial strain by determining the effect of the prophage $\phi$ Rs551, through mutagenesis, on the virulence and competitive fitness of its host strain UW551 of $R$. solanacearum.

Recently, we found that the filamentous phage $\phi$ Rs551 was stably maintained in the genome of the race 3 biovar 2 strain UW551 of $R$. solanacearum as a prophage, and released to the supernatant of the bacterial strain under normal growth condition (Ahmad et al., 2017). In addition, infection of a $\phi$ Rs551-lacking $R$. solanacearum strain RUN302 by the phage caused integration of $\phi$ Rs551 into the genome of RUN302, resulting in significantly reduced EPS production, swimming, swarming, and twitching motilities, as well as virulence (Ahmad et al., 2017). In this study, we determined the effect of $\phi$ Rs551 on its carrier $R$. solanacearum strain UW551 in virulence and competitive fitness by deleting 3,321 of $\phi$ Rs551's 7,929-bp prophage region from the UW551 genome. As expected, no phage particles were detected, since all but one structural genes of $\phi R 5551$ were deleted in the mutant (Figure 1). The deletion also resulted in significantly increased virulence as compared to the wt strain UW551, probably due to the increased EPS production and twitching motility (as indicated by the over-expression of the pilT gene), as well as the over-expression of other virulence genes including egl and pehC, and virulence regulatory genes $p h c A$ and $h r p B$. Repeated attempts to delete $\phi$ Rs551's entire 7,929-bp prophage region from UW551 were unsuccessful, suggesting that at least a portion of the phage may be essential to $R$. solanacearum UW551 for unknown reasons.

The discovery that the genome of $\phi$ Rs551was present with $100 \%$ identity in the deposited genomes of 11 race 3 biovar 2 phylotype IIB sequevar 1 strains of $R$. solanacearum isolated from different countries at different times and sequenced independently by different research groups (Ahmad et al., 2017) raised the question about the evolutionary and biological significance of the prophage in the sequevar 1 strains of 


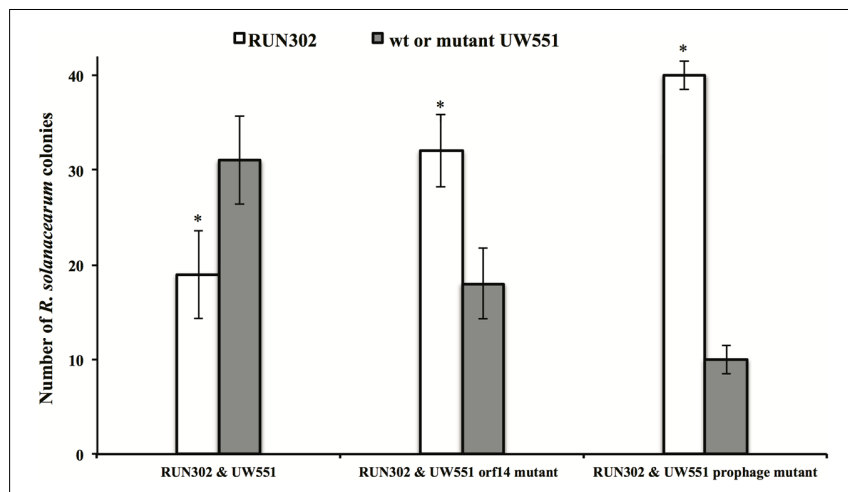

FIGURE 5 | Competitive fitness of $R$. solanacearum strains in tomato stems. Two- to three-week-old tomato plants were inoculated by soil drenching with a mixture of two different $R$. solanacearum strains as indicated $(25 \mathrm{ml}: 25 \mathrm{ml}$, $5 \times 10^{7}$ cells $\left./ \mathrm{ml}\right)$. At first sign of wilt symptoms, the population ratio of each strain was determined by serial dilution plating of ground $0.5-\mathrm{cm}$ stem, followed by a multiplex PCR (Stulberg et al., 2015) to differentiate the strains. Values are means of three experiments, each containing 50 randomly picked colonies from five wilted plants per treatment. Bars indicate standard errors. *indicates significant difference $(p<0.05)$ between RUN302 and the wt or mutant UW551 strains by the Student's $t$-test.

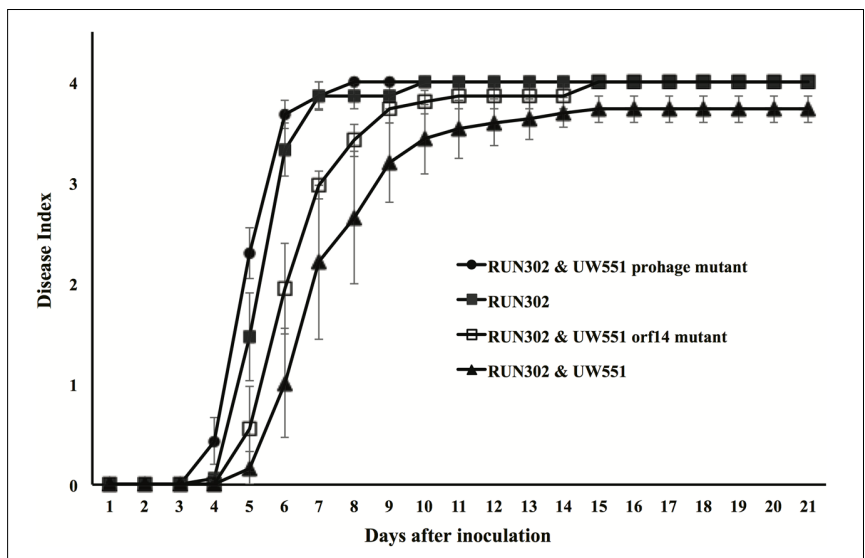

FIGURE 6 | Effect of wt or mutant UW551 strain on the virulence of RUN302 strain of $R$. solanacearum. Two- to three-week-old tomato plants were inoculated by soil drenching with $50 \mathrm{ml}\left(5 \times 10^{7}\right.$ cells $\left./ \mathrm{ml}\right)$ of RUN302 alone, or with a 1:1 mixture of two different $R$. solanacearum strains as indicated. Plants were rated using a disease index of 0 (no wilting) to 4 (76-100\% leaves wilted). Points represent means of three separate experiments with 10 plants in each experiment for a total of 30 plants. Bars indicate standard errors.

R. solanacearum. Results from our competition assay revealed that $\phi$ Rs551 may offer a competitive advantage to its lysogenic host strain UW551 by out-competing its prophage Rs551-lacking strain RUN302 in a mixed infection, since the number of UW551 colonies was significantly higher than that of the RUN302 ones in infected tomato stems only when the wt strain UW551, but not the UW551 prophage mutant, was co-inoculated with RUN302. In virulence, the wt UW551 significantly reduced the virulence of RUN302 when the two strains were co-inoculated, similar to the effect caused by infection of RUN302 by $\phi$ Rs551 (Ahmad et al., 2017). The UW551 prophage mutant, however, had little effect on the virulence of RUN302 in the mixed infection of tomato plants, when the prophage of $\phi$ Rs551 was mutated (Figure 6). The results from our previous and current studies suggest that ecologically, $\phi$ Rs551 may offer a competitive advantage to its host $R$. solanacearum by rendering the lysogenic $R$. solanacearum strain less virulent in plant hosts and more dominant over $\phi$ Rs551-lacking $R$. solanacearum strains when occupying the same niche, and therefore more persistent in the environment such as plant, soil, and water. This in turn prolongs the symbiotic relationship between $\phi$ Rs551 and UW551.

Since $\phi$ Rs551 contains a putative type-2 repressor gene orf14, it is possible that the observed physiological changes in the UW551 prophage mutant of $R$. solanacearum are due to the lack of transcriptional repression of bacterial virulence-related genes by the phage's repressor. Such a hypothesis has also been proposed by Addy et al. (2012a) for the type-1 phage repressor. Since the UW551 prophage mutant contains a mutation of orf14, we generated the UW551 orf14 mutant by deleting a 342-bp prophage region from the UW551 genome (which includes the 291-bp orf14 of $\phi R$ s551) to determine if orf14 solely played a role in the observed physiological changes in the prophage mutant. In contrast with the UW551 prophage mutant, the UW551 orf14 mutant produced phage particles like the wt strain UW551. Similar to the prophage mutant strain, however, the orf 14 mutant was significantly increased in production of EPS and expression of the virulence and virulence regulatory genes assayed. This may lead to increased virulence of the orf14 mutant as compared to the wt strain UW551, although the level of increase was not as high as the prophage mutant strain, suggesting that the observed physiological changes in the prophage mutant are at least partially caused by deletion of the orf14 gene. The partial effect of the type 2 repressor encoded by orf14 of $\phi$ Rs551 is different from the type 1 repressor encoded by orf 15 of another filamentous $R$. solanacearum phage RSM3, since the loss of virulence caused by infection with $\phi$ RSM3 can be fully restored when the ORF15 of QRSM3 was deleted (Addy et al., 2012a). This suggests that the type 2 phage repressor in $\phi$ Rs551 may not regulate virulence as tightly as the type 1 repressor in $\phi$ RSM3. Results from our competition assays revealed that like the UW551 prophage mutant, the UW551 orf14 mutant was significantly out competed by strain RUN302 in colonizing tomato stems (Figure 5), and had little effect on the virulence of RUN302 9 days after mixed infection of tomato plants (Figure 6). These results suggest that orf14 may only be partially responsible for offering competitive fitness to the wt strain UW551 in tomato stems and in reducing virulence of the $\phi$ Rs551-lacking strain RUN302 in mixed infection. This partial effect of the UW551 orf14 mutant may be explained by continued production of phage particles in the mutant, thereby exhausting the energy of the mutant or triggering other physiological changes in the mutant and/or host plant.

It is unclear why the expression of the pilT gene in the UW551 orf14 mutant was 21.1-fold higher, but the UW551 prophage mutant was only 8.4-fold higher than the wt strain. Under a microscope, the size of the corrugated trajectories around the colonies of the mutant also looked bigger in the orf14 mutant than in the prophage mutant (Figure 2). Future studies are 
needed to determine how ORF14 regulates the expression of the pilT gene and the significance of PilT in other biological functions other than twitching motility in strain UW551 of R. solanacearum.

\section{CONCLUSION}

We demonstrated that the prophage Rs551 affects multiple important physiological functions of and offers competitive fitness to its carrier $R$. solanacearum strain UW551, at least partially through the type 2 phage repressor encoded by orf14. Future research, however, is needed to determine exactly how the phage repressor regulates these functions in the bacterial strain, and what other phage factors contribute to the virulence and competiveness fitness of the carrier bacterial strain against other $\phi$ Rs551-lacking $R$. solanacearum strain occupying the same environment and competing for the same ecological niche for plant infection and survival. A better understanding of the relationship between the

\section{REFERENCES}

Addy, H. S., Askora, A., Kawasaki, T., Fujie, M., and Yamada, T. (2012a). Loss of virulence of the phytopathogen Ralstonia solanacearum through infection by $\phi$ RSM filamentous phages. Phytopathology 102, 469-477. doi: 10.1094/ PHYTO-11-11-0319-R

Addy, H. S., Askora, A., Kawasaki, T., Fujie, M., and Yamada, T. (2012b). The filamentous phage $\phi R S S 1$ enhances virulence of phytopathogenic Ralstonia solanacearum on tomato. Phytopathology 102, 244-251. doi: 10.1094/PHYTO10-11-0277

Agricultural Bioterrorism Protection Act of 2002 (2002). Possession, Use, and Transfer of Biological Agents and Toxins; Interim Rule and Request for Comments. 67 Federal Register 240. Washington, DC: United States Department of Agriculture, 76908-76938.

Ahmad, A. A., Stulberg, M. J., Mershon, J. P., Mollov, D. S., and Huang, Q. (2017). Molecular and biological characterization of $\phi R s 551$, a filamentous bacteriophage isolated from a race 3 biovar 2 strain of Ralstonia solanacearum. PLOS ONE 12:e0185034. doi: 10.1371/journal.pone.0185034

Albuquerque, G. M. R., Santos, L. A., Felix, K. C. S., Rollemberg, C. L., Silva, A. M. F., Souza, E. B., et al. (2014). Moko disease-causing strains of Ralstonia solanacearum from Brazil extend known diversity in paraphyletic phylotype II. Phytopathology 104, 1175-1182. doi: 10.1094/PHYTO-12-13-0334-R

Allen, C., Huang, Y., and Sequeira, L. (1991). Cloning of genes affecting polygalacturonase production in Pseudomonas solanacearum. Mol. PlantMicrobe Interact. 4, 147-154. doi: 10.1094/MPMI-4-147

Askora, A., Kawasaki, T., Fujie, M., and Yamada, T. (2011). Resolvase-like serine recombinase mediates integration/excision in the bacteriophage $\phi R S M$. J. Biosci. Bioeng. 111, 109-116. doi: 10.1016/j.jbiosc.2010.10.001

Askora, A., Kawasaki, T., Usami, S., Fujie, M., and Yamada, T. (2009). Host recognition and integration of filamentous phage $\phi R S M$ in the phytopathogen, Ralstonia solanacearum. Virology 384, 69-76. doi: 10.1016/j.virol.2008.11.007

Bhunchoth, A., Phironrit, N., Leksomboon, C., Chatchawankanphanich, O., Kotera, S., Narulita, E., et al. (2015). Isolation of Ralstonia solanacearuminfecting bacteriophages from tomato fields in Chiang Mai, Thailand, and their experimental use as biocontrol agents. J. Appl. Microbiol. 118, 1023-1033. doi: $10.1111 /$ jam.12763

Bohannan, B. J. M., and Lenski, R. E. (2000a). The relative importance of competition and predation varies with productivity in a model community. Am. Nat. 156, 329-340. doi: 10.1086/303393

Bohannan, B. J. M., and Lenski, R. E. (2000b). Linking genetic change to community evolution: insights from studies of bacteria and bacteriophage. Ecol. Lett. 3, 362-377. doi: 10.1046/j.1461-0248.2000.00161.x phage and the bacterium will facilitate effective control of R. solanacearum.

\section{AUTHOR CONTRIBUTIONS}

Conceived and designed the experiments: AA, MS, and QH. Performed the experiments: AA and MS. Analyzed the data: $\mathrm{AA}$ and $\mathrm{QH}$. Contributed reagents/materials/analysis tools: QH. Wrote the paper: AA and QH.

\section{ACKNOWLEDGMENTS}

This research was financially supported by the United States Department of Agriculture, Agricultural Research Service. We thank Joseph Mowery and the Beltsville Electron and Confocal Microscope Unit for technical assistance. We also thank John Hartung in the United States for critical review of our manuscript.

Brüssow, H., Canchaya, C., and Hardt, W. D. (2004). Phages and the evolution of bacterial pathogens: from genomic rearrangements to lysogenic conversion. Microbiol. Mol. Biol. Rev. 68, 560-602. doi: 10.1128/MMBR.68.3.560-602. 2004

Canchaya, C., Fournous, G., and Brüssow, H. (2004). The impact of prophages on bacterial chromosomes. Mol. Microbiol. 53, 9-18. doi: 10.1111/j.1365-2958. 2004.04113.x

Casjens, S. (2003). Prophages and bacterial genomics: what have we learned so far? Mol. Microbiol. 49, 277-300. doi: 10.1046/j.1365-2958.2003.03580.x

Davies, E. V., Winstanley, C., Fothergill, J. L., and James, C. E. (2016). The role of temperate bacteriophages in bacterial infection. FEMS Microbiol. Lett. 363:fnw015. doi: 10.1093/femsle/fnw015

Delaspre, F., Nieto Peñalver, C. G., Saurel, O., Kiefer, P., Gras, E., Milon, A., et al. (2007). The Ralstonia solanacearum pathogenicity regulator HrpB induces 3hydroxy-oxindole synthesis. Proc. Natl. Acad. Sci. U.S.A. 104, 15870-15875. doi: 10.1073/pnas.0700782104

Denny, T. P. (2006). "Plant pathogenic Ralstonia species," in Plant-Associated Bacteria, ed. S. S. Gnanamanickam (Dordrecht: Springer Publishing), 573-644. doi: 10.1007/978-1-4020-4538-7_16

Fujiwara, A., Fujisawa, M., Hamasaki, R., Kawasaki, T., Fujie, M., and Yamada, T. (2011). Biocontrol of Ralstonia solanacearum by treatment with lytic bacteriophages. Appl. Environ. Microbiol. 77, 4155-4162. doi: 10.1128/AEM. 02847- 10

Genin, S., and Boucher, C. (2004). Lessons learned from the genome analysis of Ralstonia solanacearum. Annu. Rev. Phytopathol. 42, 107-134. doi: 10.1146/ annurev.phyto.42.011204.104301

González, E. T., and Allen, C. (2003). Characterization of a Ralstonia solanacearum operon required for polygalacturonate degradation and uptake of galacturonic acid. Mol. Plant Microbe Interact. 16, 536-544. doi: 10.1094/MPMI.2003.16. 6.536

Hayward, A. C. (1991). Biology and epidemiology of bacterial wilt caused by Pseudomonas solanacearum. Annu. Rev. Phytopathol. 29, 65-87. doi: 10.1146/ annurev.py.29.090191.000433

Huang, Q., and Allen, C. (2000). Polygalacturonases contribute to colonization ability and virulence of Ralstonia solanacearum on tomato plants. Physiol. Mol. Plant Pathol. 57, 77-83. doi: 10.1006/pmpp.2000.0283

Huang, Q., and Lakshman, D. K. (2010). Effect of clove oil on plant pathogenic bacteria and bacterial wilt of tomato and geranium. J. Plant Pathol. 92, 701-707.

Huerta, A. I., Milling, A., and Allen, C. (2015). Tropical strains of Ralstonia solanacearum outcompete race 3 biovar 2 strains at lowland tropical temperatures. Appl. Environ. Microbiol. 81, 3542-3551. doi: 10.1128/AEM. 04123-14 
Joo, J., Gunny, M., Cases, M., Hudson, P., Albert, R., and Harvill, E. (2006). Bacteriophage-mediated competition in Bordetella bacteria. Proc. R. Soc. Lond. B Biol. Sci. 273, 1843-1848. doi: 10.1098/rspb.2006. 3512

Kang, Y., Liu, H., Genin, S., Schell, M. A., and Denn, T. P. (2002). Ralstonia solanacearum requires type 4 pili to adhere to multiple surfaces and for natural transformation and virulence. Mol. Microbiol. 2, 427-437. doi: 10.1046/j.13652958.2002.03187.x

Kawasaki, T., Nagata, S., Fujiwara, A., Satsuma, H., Fujie, M., Usami, S., et al. (2007a). Genomic characterization of the filamentous integrative bacteriophages $\phi \mathrm{RSS} 1$ and $\phi \mathrm{RSM} 1$, which infect Ralstonia solanacearum. J. Bacteriol. 189, 5792-5802.

Kawasaki, T., Narulita, E., Matsunami, M., Ishikawa, H., Shizu, M., Fujie, M., et al. (2016). Genomic diversity of large-plaque-forming podoviruses infecting the phytopathogen Ralstonia solanacearum. Virology 492, 73-81. doi: 10.1016/j. virol.2016.02.011

Kawasaki, T., Satsuma, H., Fujie, M., Usami, S., and Yamada, T. (2007b). Monitoring of phytopathogenic Ralstonia solanacearum cells using green fluorescent protein-expressing plasmid derived from bacteriophage $\phi$ RSS1. J. Biosci. Bioeng. 104, 451-456. doi: 10.1263/jbb.104.451

Kawasaki, T., Shimizu, M., Satsuma, H., Fujiwara, A., Fujie, M., Usami, S., et al. (2009). Genomic characterization of Ralstonia solanacearum phage ?RSB1, a T7-like wide-host-range phage. J. Bacteriol. 191, 422-427. doi: 10.1128/JB. 01263-08

Kelman, A. (1954). The relationship of pathogenicity in Pseudomonas solanacearum to colony appearance on a tetrazolium medium. Phytopathology 64, 693-695.

Kelman, A., Hartman, G. L., and Hayward, A. C. (1994). "Introduction," in Bacterial Wilt: the Disease and its Causative Agent, Pseudomonas solanacearum, eds A. C. Hayward and G. L. Hartman (Wallingford: CAB International), $1-7$.

Koskella, B., and Brockhurst, M. A. (2014). Bacteria-phage coevolution as a driver of ecological and evolutionary processes in microbial communities. FEMS Microbiol. Rev. 38, 916-931. doi: 10.1111/1574-6976.12072

Koskella, B., Lin, D. M., Buckling, A., and Thompson, J. N. (2012). The costs of evolving resistance in heterogeneous parasite environments. Proc. R. Soc. Lond. B Biol. Sci. 279, 1896-1903. doi: 10.1098/rspb.2011.2259

Liu, H., Kang, Y., Genin, S., Schell, M. A., and Denny, T. P. (2001). Twitching motility of Ralstonia solanacearum requires a type IV pilus system. Microbiology 147, 3215-3229. doi: 10.1099/00221287-147-12-3215

Livak, K. J., and Schmittgen, T. D. (2001). Analysis of relative gene expression data using real-time quantitative PCR and the $2^{-} \Delta \Delta^{C_{\mathrm{TM}}}$ method. Methods 25, 402-408. doi: 10.1006/meth.2001.1262

Miller, J. H. (1972). Experiments in Molecular Genetics. Cold Spring Harbor, NY: Cold Spring Harbor Laboratory.

Murugaiyan, S., Bae, J. Y., Wu, J., Lee, S. D., Um, H. Y., Choi, H. K., et al. (2011). Characterization of filamentous bacteriophage PE226 infecting Ralstonia solanacearum strains. J. Appl. Microbiol. 110, 296-303. doi: 10.1111/j. 1365-2672.2010.04882.x

Ozawa, H., Tanaka, H., Ichinose, Y., Shiraishi, H., and Yamada, T. (2001). Bacteriophage P4282, a parasite of Ralstonia solanacearum, encodes a bacteriolytic protein important for lytic infection of its host. Mol. Genet. Genomics 265, 95-101. doi: 10.1007/s004380000389
Poteete, A. R., Rosadini, C., and Pierre, C. (2006). Gentamicin and other cassettes for chromosomal gene replacement in Escherichia coli. Biotechniques 41, 261-264. doi: 10.2144/000112242

Roberts, D. P., Denny, T. P., and Schell, M. (1988). Cloning of the egl gene of Pseudomonas solanacearum and analysis of its role in pathogenicity. J. Bacteriol. 170, 1445-1451. doi: 10.1128/jb.170.4.1445-1451.1988

Sambrook, J., and Russell, D. W. (2001). Molecular Cloning: a Laboratory Manual, 3rd Edn. Cold Spring Harbor, NY: Cold Spring Harbor Laboratory Press.

Schell, M., Roberts, D. P., and Denny, T. P. (1988). Analysis of the Pseudomonas solanacearum polygalacturonase encoded by $\mathrm{pglA}$ and its involvement in phytopathogenicity. J. Bacteriol. 170, 4501-4508. doi: 10.1128/jb.170.10.45014508.1988

Schell, M. A. (2000). Control of virulence and pathogenicity genes of Ralstonia solanacearum by an elaborated sensory array. Annu. Rev. Phytopathol. 38, 263-292. doi: 10.1146/annurev.phyto.38.1.263

Stulberg, M. J., and Huang, Q. (2015). A TaqMan-based multiplex qPCR assay and DNA extraction method for phylotype IIB sequevars 1\&2 (Select Agent) strains of Ralstonia solanacearum. PLOS ONE 10:e0139637. doi: 10.1371/journal.pone. 0139637

Stulberg, M. J., and Huang, Q. (2016). A computer program for fast and easy typing of a partial endoglucanase gene sequence into phylotypes and sequevars $1 \& 2$ (select agents) of Ralstonia solanacearum. J. Microbiol. Methods 123, 101-107. doi: 10.1016/j.mimet.2016.02.010

Stulberg, M. J., Shao, J., and Huang, Q. (2015). A multiplex PCR assay to detect and differentiate select agent strains of Ralstonia solanacearum. Plant Dis. 99, 333-341. doi: 10.1094/PDIS-05-14-0483-RE

Thi, B. V. T., Khanh, N. H. P., Namikawa, R., Miki, K., Kondo, A., Thi, P. T. D., et al. (2015). Genomic characterization of Ralstonia solanacearum phage RS138 of the family Siphoviridae. Arch. Virol. 161, 483-486. doi: 10.1007/s00705-0152654- 1

Van, T. T. B., Yoshida, S., Miki, K., Kondo, A., and Kamei, K. (2014). Genomic characterization of ?RS603, a filamentous bacteriophage that is infectious to the phytopathogen Ralstonia solanacearum. Microbiol. Immunol. 58, 697-700. doi: 10.1111/1348-0421.12203

Vasse, J., Genin, S., Frey, P., Boucher, C., and Brito, B. (2000). The hrpB and hrpG regulatory genes of Ralstonia solanacearum are required for different stages of the tomato root infection process. Mol. Plant Microbe Interact. 13, 259-267. doi: 10.1094/MPMI.2000.13.3.259

Yamada, T., Kawasaki, T., Nagata, S., Fujiwara, A., Usami, S., and Fujie, M. (2007). New bacteriophage that infect the phytopathogen Ralstonia solanacearum. Microbiology 153, 2630-2639. doi: 10.1099/mic.0.2006/ 001453-0

Conflict of Interest Statement: The authors declare that the research was conducted in the absence of any commercial or financial relationships that could be construed as a potential conflict of interest.

Copyright (c) 2017 Ahmad, Stulberg and Huang. This is an open-access article distributed under the terms of the Creative Commons Attribution License (CC BY). The use, distribution or reproduction in other forums is permitted, provided the original author(s) or licensor are credited and that the original publication in this journal is cited, in accordance with accepted academic practice. No use, distribution or reproduction is permitted which does not comply with these terms. 